\title{
Prevalence and Intensity of Soil-Transmitted Helminths Among School-Aged Children in Sigmo Primary School, Jimma Zone, South-Western Ethiopia
}

\author{
Daniel Emana*, Kalid Jemal, Mitiku Bajiro, Zeleke Mekonnen \\ Department of Medical Laboratory Sciences and Pathology, College of Health Sciences, Jimma University, Jimma, Ethiopia
}

\section{Email address:}

daniel.emana@ju.edu.et (D.Emana),kalidjemal4@gmail.com (K. Jemal), mitikubajiro2008@yahoo.com (M. Bajiro), zeleke.mekonnen@ju.edu.et (Z. Mekonnen)

\section{To cite this article:}

Daniel Emana, Kalid Jemal, Mitiku Bajiro, Zeleke Mekonnen. Prevalence and Intensity of Soil-Transmitted Helminths Among School-Aged Children in Sigmo Primary School, Jimma Zone, South-Western Ethiopia. Clinical Medicine Research. Vol. 4, No. 4, 2015 , pp. 98-103. doi: $10.11648 /$ j.cmr.20150404.12

\begin{abstract}
Background: School-aged children have been identified as high risk group of population to be infected with soiltransmitted helminths (STH) in developing countries. Although, there were previous reports concerning the prevalence; but limited data are available with regards to intensity of the STH. Therefore, the objective of current study was to assess the prevalence and intensity of STH and associated risk factors among school-aged children at Sigmo primary school. Method: Cross-sectional study design was conducted from March-April 2015, at Sigmo primary school children, Jimma Zone, Ethiopia. Study subjects were selected from grade 1-8 with in age groups between 5-18 yrs old with multistage sampling techniques. The demographic and risk factor analysis data were collected by using pre-tested and structured questionnaires from parents/guardian or teachers of the children. Prevalence and intensity of STH were determined by using Kato-Katz thick smear techniques. Student's data were analyzed by SPSS version 20 software package, both logistic regression and chi-square statistical analysis approaches were applied. Results: Out of 330 calculated sample sizes, 302 were participated in the study. The overall prevalence of STH was 41.7\%. A. lumbricoides was the predominant parasite (19.8\%) followed by T. trichiura (15.6\%). In logistic regression, latrine usage (AOR: 1.77, 95\% $\mathrm{CI}, 1.09-2.87, \mathrm{P}=0.020)$, habit of hand washing before meal $(\mathrm{AOR}=1.776,95 \% \mathrm{CI}$, 1.098-2.871, $\mathrm{P}=0.019)$ and habit of hand washing after toilet ( $\mathrm{AOR}=1.595,95 \% \mathrm{CI}, 1.005-2.531, \mathrm{P}=0.048)$ were predictor of STH infections in the study area. Regarding sex as possible risk factor, male children were infected more than female children, but not statistical significant. Overall infection intensity of $A$. lumbricoides in the study area was (20.2\%), T. trichiura (16.9\%) and hookworm was (1.67\%), respectively. Conclusion: Prevalence of STHs in the study area was quite high and calls for at least annual mass drug administration in addition to prompting preventive actions like health education, personal hygiene and provision of clean water in the study area.
\end{abstract}

Keywords: Prevalence, STH, Sigmo Primary School, School-Aged Children

\section{Background}

Soil-transmitted helminths (STHs) have been among the major chronic parasitic infections distributed throughout the world. Globally, about 4.5 billion individuals are at risk and more than 1 billion peoples become infected, of which about 450 million suffer from the infection of which most of them are school-aged children[1, 2]. An estimation of the STHs cases in number indicated A. lumbricoides, T. Trichiura and Hookworm species were 1,121, 795 and 740 million, respectively. In addition to mortality and morbidity progresses, the parasitic infections have been known to cause stunting growth, poor appetites, poor physical fitness and cognitive effects on school-aged children[3, 4].

The occurrences and distribution of STH infection is directly associated with factors such as: socioeconomic status of the community, poor sanitation of environment and lack of awareness about personal hygiene, latrine usage, lack of access to clear water and climatic changes [5]. School-aged children, pregnant women and preschool-aged children are high risk group for infectious diseases and STH infections [3, 6, 7]. Moreover, factors such as lack of competent immune system, development of protective personal hygiene and nutritional supplements also contribute for those infections [7-9]. 
Infection with STH such as: A. lumbricoides, $T$. trichiura and Hookworm are more common in school-aged children in Ethiopia and they suffer more from multiple infections. Previous epidemiological surveys have drawn attention to importance of intensity of the intestinal helminths infection with respect to morbidity they pose and necessity for sustainable control [10].

It has been frequently reported concerning prevalence of STHs and other intestinal parasites in different part of Ethiopia among school-aged children, but there is no as such organized and published report on infection intensity of STHs on the school-aged children [11-13]. Moreover, prevalence varies extremely from place to place which in turn influences the administration of preventive chemotherapy. Therefore, the aims of current study were to determine prevalence of intestinal parasites, identify associated possible risk factors and infection intensity among school-aged children at Sigmo woreda, Jimma zone.

\section{Methods}

\subsection{Study Area and Study Design}

A cross sectional study design was conducted to determine the prevalence and intensity of STH among primary school-aged children in Sigmo district, Jimma Zone, Oromia Region, Ethiopia in 2015. The town is located at $474 \mathrm{~km}$ to south-west from Addis Ababa. The town altitude laid between $1,672-1,864$ meter and latitudes of $7^{\circ} 42^{\prime} 50^{\prime \prime} \mathrm{N}$ and $07^{\circ} 53^{\prime} 50 " \mathrm{~N}$ and between longitudes $37^{\circ} 11^{\prime} 22^{\prime \prime} \mathrm{E}$ and $37^{\circ} 20^{\prime} 36^{\prime \prime} \mathrm{E}$, above sea level. The study area has warm to hot climate and sub-humid, and having 1,300-1,800 mm annual rain fall, with temperature range of $20-30^{\circ} \mathrm{C}$. The pattern of rain fall in study area has been similar to other parts of Ethiopia with long rainy season from June to September whereas short rainy season begins from March to May. Approximate population size in the study area according to 2007 census data was about 114,000.

All students willing to participate and where their parents/guardians consent were included, but students with diarrhea and/or unwilling to participate were excluded from the study.

\subsection{Sample Size and Sampling Techniques}

Sample size was calculated using single population proportion formula estimate considering 5\% level of the significances and $68.7 \%$ prevalence of the STH infection in Jimma Zone [14]. Using the formula at 95\% confidence interval $(\mathrm{CI})$, the sample size was calculated as $\mathrm{n}=\left[\mathrm{Z}_{1-\alpha / 2}\right]^{2} \mathrm{P}$ $(1-p] / d^{2}$. Where: $(Z(1-\alpha ́ / 2)=1.96)$ of $95 \% \mathrm{CI}, \mathrm{n}=$ sample size, $\mathrm{P}=$ proportion problem in the study area, $\mathrm{d}=$ Marginal error to be tolerated $(5 \%)$. Multistage sampling technique was employed to select the study students. Classes were first selected by using simple random sampling technique and study subject was sampled, then the sampled children were selected by using systematic random sampling using student class roster (attendance) in the year of study as sampling frame. Thus, proportional allocation was employed to select study subject from each selected section of the students.

However, of the 330 total sample size calculated, only 302 children were included in the final analysis as some samples were not sufficient for microscopy, and others showed unwillingness to participate.

\subsection{Parasitological Examinations}

After socio-demographic data was collected from selected students, labeled stool plastic cup-capped well bearing serial numbers of the study subject was given. The students requested to put about 5 grams of fresh stool into the stool container. Stool samples were processed using $0.85 \%$ normal saline for direct smear and identification of motile intestinal parasites. From the remaining sample Kato-Katz thick smear technique was processed [3]. The slides were examined under light microscope at $100 \mathrm{X}$ and $400 \mathrm{X}$ magnifications. Total STH eggs count on the slides were taken and converted in to egg per gram (epg) of stool by multiplying with a factor of 24 to evaluate the intensity of infection.

Finally, the intensity of the infection was estimated based on cut-off value for the classification of infection intensity of the eggs per gram of stool as follows: for A. lumbricoides: light infection (1-4,999 epg), moderate infection (5000-49,999 epg) and heavy infection (above 50,000 epg); for T. trichiura: light infection (1-999 epg), moderate infection (1000-9,999 epg) and heavy infection (above 10,000 epg); for hookworms: light infection (1-1,999 epg), moderate infection (2000-3,999 epg) and heavy infection (above 4000 epg) [15].

\subsection{Quality Control}

Pre-test was conducted to check the structure of our questionnaires on small number of students. The obtained data consistence was checked at the organized center for data collection and examination. All laboratory reagents, materials, process of transportation and functionality of microscopy at the center were checked by senior laboratory professional. Code of sample was cross checked with study subject number. Kato-katz thick smear technique was performed at field, to avoid observational bias each slide was re-checked by senior laboratory technologists from Jimma University.

\subsection{Data Analysis}

The obtained data were entered, cleaned for relevant information and analyzed by using SPSS (Version 20) software package. Socio-demographic profiles of the school-aged children were summarized by descriptive statistics. Logistic regression analysis was used to determine and study the association between explanatory variable (independent variable) and outcome (dependant variable). Adjusted odd ratio (AOR), 95\% Confidence interval and $\mathrm{P}$-value $<0.05$ was used to assess the magnitude of association and their significance level.

\subsection{Ethical Consideration}

Ethical clearance was obtained from Jimma University, College of Health Sciences ethical review board. Additionally, 
after explaining the importance of the study briefly, an informed written consent was obtained from parents/ guardians of the students and confidentiality of the study participants was also maintained. Those students, who become positive for any STHs, were treated with single dose albendazole $400 \mathrm{mg}$ tablets. For other intestinal parasitic infections children were referred to nearby health center for appropriate treatments.

\section{Results}

\subsection{Socio-Demographic Characteristics of Sigmo Elementary School-Aged Children}

A total of 302 school-aged children were included in the current study, of which male accounts $47.7 \%$ (144/302) and female $52.3 \%$ (158/302). Age ranges of the students were 6 to 18years with mean age of $11.03( \pm 2.5 \mathrm{SD})$ years old. Habit of latrine usage 'Always' 201 (66.6\%) 'Sometimes' 101 (33.4\%), habit of hand washing before meal 'Always' 118 (39.1\%) 'Sometimes' $184(60.9 \%)$, habit of hand washing after toilet 'Always' 145 (48.0\%) 'Sometimes' 157 (52.0\%), habit of finger nail trimming 'Always' 191 (63.2\%) 'Sometimes' 111 (33.8\%) and habit of shoe wearing was 'always' 299 (99\%) whereas 'sometimes' 3 (1\%).

Among 302 study subject significant association was observed in Latrine usage $\left(X^{2}: 5.43 ; p=0.02\right)$, Habit of hand washing before meal $\left(X^{2}: 5.53 ; p=0.019\right)$, Habit of hand washing after toilet $\left(X^{2}: 3.94 ; p=0.047\right)$. But, there were no significant association observed among gender, age category and habit of finger nail trimming status.

Table 1. Prevalence of STH in association of potential risk factors among School-aged children, Sigmo Elementary School, Jimma Zone, March 2015.

\begin{tabular}{|c|c|c|c|c|c|}
\hline Variable & & $\begin{array}{l}\text { Total examined } \\
\text { n (\%) }\end{array}$ & $\begin{array}{l}\text { Positives } \\
\text { n (\%) }\end{array}$ & $X^{2}$ & $P$-value $(\mathrm{CI}=95 \%)$ \\
\hline \multirow{2}{*}{ Gender } & Male & $144(47.7)$ & $62(43.1)$ & 0.201 & 0.65 \\
\hline & Female & $158(52.3)$ & $64(40.5)$ & & \\
\hline \multirow{3}{*}{ Age category } & $5-10$ & $140(46.4)$ & $57(40.7)$ & 0.631 & 0.73 \\
\hline & $11-15$ & $158(52.3)$ & $68(43.04)$ & & \\
\hline & $16-20$ & $4(1.3)$ & $1(25)$ & & \\
\hline \multirow{2}{*}{ Latrine Usage } & Always & $201(66.7)$ & $52(25.9)$ & 5.43 & 0.02 \\
\hline & Sometimes & $101(33.3)$ & $74(73.3)$ & & \\
\hline \multirow{2}{*}{ HWBM } & Always & $118(39.1)$ & $39(33.1)$ & 5.53 & 0.019 \\
\hline & Sometimes & $184(60.9)$ & $87(47.3)$ & & \\
\hline \multirow{2}{*}{ HWAT } & Always & $145(48)$ & $52(35.9)$ & 3.94 & 0.047 \\
\hline & Sometimes & $157(52)$ & $74(47.1)$ & & \\
\hline \multirow{2}{*}{ FNTH } & Always & $191(63.2)$ & $52(27.2)$ & 2.2 & 0.14 \\
\hline & Sometimes & $111(36.8)$ & 74 (66.7) & & \\
\hline
\end{tabular}

Keys: HWBM: hand washing habit before meal, HWAT: Hand washing habit after toilet; FNTH: Finger nails trimming habit , $\mathrm{X}^{2}$ : Chi Square

\subsection{Parasitological Findings of Prevalence and Intensity of Soil-Transmitted Helminths}

Among 302 school children 126 (41.7\%) of them become positive for at least one of STHs by Kato Katz thick smear techniques. Among STHs identified in this study, $A$. lumbricoides was the predominant parasite $60(19.8 \%)$ followed by T. trichiura 47 (15.6\%) and Hookworm species 3 (1\%). Other intestinal parasites identified during examination includes; S. stercolaris, E. histolytica/dispar and others $(G$. lamblia and Taenia species) 7 (2.3\%), 6 (2\%) and 3 (1\%), respectively (Table 2).

Table 2. Identified STH with respect to gender of students at Sigmo Elementary School, Jimma Zone, March 2015.

\begin{tabular}{|c|c|c|c|c|c|}
\hline \multirow{2}{*}{$\begin{array}{l}\text { Parasites } \\
\text { identified }\end{array}$} & \multirow{2}{*}{$\begin{array}{l}\text { Male }(n=144) \\
n(\%)\end{array}$} & \multirow{2}{*}{$\begin{array}{l}\text { Female }(n=158) \\
n(\%)\end{array}$} & \multirow{2}{*}{$\begin{array}{l}\text { Total }(n=302) \\
n(\%)\end{array}$} & \multirow{2}{*}{$\mathbf{X}^{2}$} & \multirow{2}{*}{ P.value } \\
\hline & & & & & \\
\hline A. lumbricoides & $31(21.5)$ & $29(18.6)$ & $60(19.8)$ & 0.59 & 0.44 \\
\hline T. trichiura & $21(14.6)$ & $26(16.5)$ & $47(15.6)$ & 0.21 & 0.65 \\
\hline S. stercolaris & $6(4.2)$ & $1(0.63)$ & $7(2.32)$ & & \\
\hline Hookworm & $2(1.4)$ & $1(0.63)$ & $3(1)$ & & \\
\hline E. histolytica/dispar & $2(1.4)$ & $4(2.53)$ & $6(2)$ & & \\
\hline Others & $0(0)$ & $3(1.9)$ & $3(1)$ & & \\
\hline
\end{tabular}

Chi-square and P-values were not calculated for figures less than 5.

Comparing overall prevalence of intestinal parasites between male and female, high prevalence (43.1\%) was observed among male participants compared to female participants $(40.5 \%)$. Furthermore, looking at individual parasites prevalence, A. lumbricoides was higher in male
(21.5\%) compared to female (18.6\%), where as prevalence of T. trichiura was higher in female (16.5\%) compared to male $(14.6 \%)$. On the other hand, Hookworm and S. stercolaris were also observed higher in males than in females (Table 2). Nevertheless, the observed differences between males and 
females were not significant statistically $\left(\mathrm{X}^{2}: 0.59 ; P: 0.44\right.$ for A. lumbricoides and $\mathrm{X}^{2}: 0.21 ; P: 0.65$ for T trichiura).

\subsection{Infection Intensity of STHs}

The mean egg per gram (epg) of A. lumbricoides, $T$.trichiura and Hookworm infection observed were $1,879.8$ epg (Range: 48 to 23, 980), 1,833.9 epg (Range: 24 to 16,800 ) and 273.6 epg (Range: 48 to 600), respectively. Out of 61 (20.2\%) school-aged children positive for $A$. lumbricoides $0.7 \%$ had moderate intensity infection while $19.5 \%$ had light intensity infection. Out of 53 (16.9\%) school-aged children positive for $T$. trichiura $9.6 \%$ had light intensity infection, while $6.6 \%$ had moderate intensity infection and $0.7 \%$ had heavy intensity of infection. The intensity of hookworm infection in all children was light (Table 3).

Table 3. Infection intensity of the STH among School-aged children of Sigmo Elementary School, Jimma Zone, March 2015.

\begin{tabular}{lllllll}
\hline Infection & \multicolumn{2}{c}{$\boldsymbol{A}$. lumbricoides } & \multicolumn{2}{c}{ T. trichiura } & \multicolumn{2}{c}{ Hookworm } \\
\cline { 2 - 7 } Intensity & $\mathbf{n}$ & $\%$ & $\mathbf{n}$ & $\%$ & $\mathbf{n}$ & $\%$ \\
\hline Light & 59 & $19.5 \%$ & 29 & $9.6 \%$ & 5 & $1.66 \%$ \\
Moderate & 2 & $0.70 \%$ & 20 & $6.6 \%$ & 0 & $0 \%$ \\
Heavy & 0 & $0 \%$ & 2 & $0.70 \%$ & 0 & $0 \%$ \\
Total & 61 & $20.2 \%$ & 53 & $16.9 \%$ & 5 & $1.67 \%$ \\
Positive & & & & & & \\
Negative & 241 & $79.8 \%$ & 249 & $83.1 \%$ & 297 & $98.3 \%$ \\
Total & 302 & $100 \%$ & 302 & $100 \%$ & 302 & $100 \%$ \\
\hline
\end{tabular}

\subsection{Bivariate Analysis of Risk Factors for STH Infection Among Sigmo Elementary School-Aged Children}

In present study, statistically significant association was observed between the infection status and some of risk factors (latrine usage, hand washing before meal and hand washing after toilet usage). The prevalence of STHs infection among study participants who experienced 'always' hand washing habit before meal and after toilet were $33.1 \%$ and $35.9 \%$, respectively. Whereas, who do not wash their hands either 'always' or only 'sometimes' before meal and after toilet had a prevalence of $47.3 \%$ and $47.1 \%$ STH infections, respectively. In the same way, among study participants who become positive for any STH infections and had habit of latrine use 'always', the prevalence was $25.9 \%$ compared to $73.3 \%$ who uses latrine sometimes.

The bivariate analysis of the data indicated that students who had habit of proper and regular use of latrine were less likelihood to be infected by STH infections, when compared to those only use latrine 'sometimes' (AOR: 1.77, 95\% CI, 1.09-2.87, $p=0.020$ ); students who 'always' wash their hands before meal and after toilet were also protected from being infected by STH infection than students who wash their hands 'sometimes' (AOR=1.776, 95\% CI, 1.098-2.871, $p=0.019)$, $(\mathrm{AOR}=1.595,95 \% \mathrm{CI}, 1.005-2.531, p=0.048)$, respectively.

But, other variables such as sex, age and habit of finger nail trimming were not observed as statistical significant $(p>0.05)$ (Table 4).

Table 4. Bivariate analysis of risk factors for STH among School-aged children of Sigmo Elementary School, Jimma Zone, March 2015.

\begin{tabular}{|c|c|c|c|c|c|}
\hline Variable & & Total number n (\%) & $\begin{array}{l}\text { Positive } \\
\text { n (\%) }\end{array}$ & $\operatorname{AOR}(95 \%$ CI $)$ & p-value \\
\hline \multirow{2}{*}{ Gender } & Male & $144(47.7)$ & $62(43.1)$ & $1.1(0.70-1.76)$ & 0.654 \\
\hline & Female & $158(52.3)$ & $64(40.5)$ & $\mathrm{R}$ & \\
\hline \multirow{3}{*}{ Age category } & $5-10$ & $140(46.4)$ & $57(40.7)$ & $0.483(0.05-4.8)$ & 0.536 \\
\hline & $11-15$ & $158(52.3)$ & $68(43.04)$ & $0.441(0.05-.33)$ & 0.483 \\
\hline & $16-20$ & $4(1.3)$ & $1(25)$ & $\mathrm{R}$ & \\
\hline \multirow{2}{*}{ Latrine usage } & Always & $201(66.6)$ & $52(25.9 \%)$ & $\mathrm{R}$ & \\
\hline & Sometimes & $101(33.4)$ & $74(73.3)$ & $1.77(1.09-2.87)$ & 0.02 \\
\hline \multirow{2}{*}{ HWBM } & Always & $118(39.1)$ & $39(33.1)$ & $\mathrm{R}$ & \\
\hline & Sometimes & $184(60.9)$ & $87(47.3)$ & $1.78(1.1-2.9)$ & 0.019 \\
\hline \multirow{2}{*}{ HWAT } & Always & $145(48)$ & $52(35.9)$ & $\mathrm{R}$ & \\
\hline & Sometimes & $157(52)$ & $74(47.1)$ & $1.6(1.0-2.5)$ & 0.048 \\
\hline \multirow{2}{*}{ FNTH } & Always & $191(63.2)$ & $52(27.2)$ & $\mathrm{R}$ & \\
\hline & Sometimes & $111(36.8)$ & $74(66.7)$ & $1.43(0.89-2.3)$ & 0.14 \\
\hline
\end{tabular}

Keys: HWBM: hand washing habit before meal, HWAT: Hand washing habit after toilet; FNTH: Finger nails trimming habit

\section{Discussions}

Soil-transmitted helminths infections have been the most prevalent infection among peoples living in resource limited developing countries. Despite the fact that deworming program has been on progress in most Ethiopian primary schools, since 2003 at rural area and 2009 at urban area [16], the burden of parasitic infection still becoming public health problem as revealed by this study where the overall prevalence of STH in present study area was higher than the national figures..
The results of current study revealed that the prevalence of STH among school-aged children was $41.6 \%$. This result is comparable with the previous finding from Jimma Zone, which reported $47.1 \%$ [17]. However, it was higher than the study finding in different parts of world and Ethiopia as well, Iran (19.3\%), Ujjain Madhya Pradesh, India (21.4\%), Babile Somalia region, Ethiopia (27.2\%) [18-21]. In contrast, the overall prevalence in current study was lower when compared with other studies like from Laos Southern East Asian (61.9\%), Northern East Korea (54.2\%), Southern west Ethiopia (57.4\%), Asendabo, Jimma zone (59\%) [17, 18, 22, 23]. The 
differences among the study findings might be due to differences in geographical location, climate change, living standards and socio-demographic characteristics of the populations. It had been indicated that prevalence of STHs was higher among a government/public schools compared to privately-owned schools [24] This might be also a reason why our finding was higher than some studies in Jimma zone from private schools as our study set up was also a public school where relatively poor students are attending..

In current study the prevalence of intestinal parasitic infection was slightly higher in male students (43.1\%) compared to female students, $(40.5 \%)$ although the differences were not significant statistically. This is in agreement with other studies done in Zarima town, northwest Ethiopia and Umolante district, South Ethiopia [3, 25]. This slight difference of STH infection between male and female could be explained with the fact that boys might spend more time doing outdoor activities than girls that may increase their exposure to source of infections.

Age of the children could be taken also as an important risk factor for the occurrences of intestinal parasitic infections. In the present study, relatively higher parasitic infection was observed in children with in age range of 5-10yrs old (40.7\%) and $11-15$ yrs old (43.0\%). But, children aged above 16 yrs old had low prevalence (25\%). This could be explained by the fact that this phenomenon probably might reflect age related change in exposure to STHs infection. Children at lower age have less awareness to prevent from contamination; whereas older children have an experience to keep their relative personal hygiene. In contrast other study showed the prevalence was higher in older children than younger children.

Among associated risk factors, hand washing habit before meal and toilet, and latrine usage habit of the children were the major factor among school-aged children in the study area. The odds ratio revealed that hand washing habit before meal and after toilet, and proper usage of toilet were protective from being infected by STH infection. Our results were in agreement with other studies from other parts of Ethiopia [26, 27]. This highlighted the need for integrated control of STH, i.e. deworming should be backed-up with health education to prevent re-infection. Because, the only way of re-infection with STH is exposure to the infective stages from contaminated environment as these parasites do not multiply within the human host

With respect to the intensity of $A$. lumbricoides in the current study area, majority were light intensity (19.5\%) and only few moderate $(0.7 \%)$ and no heavy intensity infection, which was nearly similar with $T$. trichiura and our findings were in agreement with other similar studies in the same zone $[6,28]$.

In Ethiopia due to poverty, low level of environmental sanitation and ignorance of simple health promotion; the diseases remain the most serious public health problem resulting in mal-absorption, diarrhea, and blood loss with consequence of anemia, poor working capacity, poor cognitive development among school-aged children and growth rate, altogether increasing the cost of drugs and morbidity rates.

\section{Conclusion and Recommendation}

Soil transmitted helminthes are the major public health problem among Sigmo primary school-aged children, where the prevalence and intensity of infections is at alarming rate. Even though deworming program for school-aged children have been launched by the government of Ethiopia, it is still at its early stage and the effectiveness of the program is far from expectations. In conclusion, the present study showed that STH was a public health problem among school-aged children in the study area necessitating annual deworming to control morbidities associated with STHs. Poor personal hygiene like not washing hands before meal and not regularly using latrine were the key factors significantly associated with STH infections among the children. This highlighted the need for integrated control of STH in the study area. Therefore, the existing health education program should be strengthened to sustain the effect of deworming as well attempt should be taken to provide safe clean water and improve the environmental sanitation and personal hygiene.

\section{Authors' Contributions}

DE, Conception of the research idea, designing data collection, analysis and interpretation of data, and finally drafting the manuscript. $\mathrm{KJ}, \mathrm{MB}$ and $\mathrm{ZM}$ involved in designing, collection of data and interpreting results and manuscript revising. All authors read and approved the final manuscript.

\section{Acknowledgements}

We would like to acknowledge Jimma University, College of health sciences for financial and material support; Sigmo school teachers for facilitating the data collection and overall coordination. We also extend our gratitude to Sigmo kebele administrative and health extension workers who collaborated for the accomplishment of this study. Finally, we would like to thank study participants, their parents/guardians for their willingness and cooperation for taking part in the study.

\section{References}

[1] WHO, Control of tropical diseases. . WHO, Geneva. 1998.

[2] WHO, Soil transmitted helminths. Fact sheet. 2014; p. p. 366.

[3] Alemu A, Asmamaw A, Addis Z, Shiferaw Y, Teklu T, Mathewos B, Wubet B, Simon G, Baye G, Soil transmitted helminths and schistosoma mansoni infections among school children in Zarima town, northwest Ethiopia. BMC infectious diseases. 2011. 11(1): p. 189.

[4] WHO, Deworming for health and development: report of the Third Global Meeting of the Partners for Parasite Control. 2005.

[5] WHO, The prevention and control of schistosomiasis and soil transmitted Helminthiasis.Geneva, World health organization.2002. 
[6] Dana D, Zeleke M, Daniel E, Miyo A, Mestawet G, Netsanet W, Josef V, Bruno L. Prevalence and intensity of soil-transmitted helminth infections among pre-school age children in 12 kindergartens in Jimma Town, southwest Ethiopia. Transactions of The Royal Society of Tropical Medicine and Hygiene. 2014; p. tru178.

[7] Getachew M, Tafesse K, Zeynudin A, Yewhalaw D, Prevalence Soil Transmitted Helminthiasis and malaria co-infection among pregnant women and risk factors in Gilgel Gibe dam Area, Southwest Ethiopia. BMC research notes. 2013; 6(1): p. 263.

[8] Quihui-Cota L, Valencia M.E, Crompton D.W.T, Philips S, Diaz Camacho S.P, Triana Tejas A. Prevalence and intensity of intestinal parasitic infections in relation to nutritional status in Mexican schoolchildren. Transactions of the Royal Society of Tropical Medicine and Hygiene. 2004; 98: p. 5.

[9] Khanal L, Choudhury DR, Rai SK, Sapkota J, Barakoti A, Amatya R, Hada S. Prevalence of intestinal worm infestations among school children in Kathmandu, Nepal. Nepal Med Coll J. 2014; 13(4): p. 272-274.

[10] Leykum., J., comparative prevalence some common intestinal helminthes infection in different attitudinal region in Ethiopia. EMJ. 1998; 36(1): p. 8-11.

[11] Gyorkos, T.W., et al., Trichuris and hookworm infections associated with anaemia during pregnancy. Tropical Medicine \& International Health.2011; 16(4): p. 7.

[12] Degarege A, E.B. Prevalence of Intestinal Parasitic Infections among Children under Five Years of Age with Emphasis on Schistosoma mansoni in Wonji Shoa Sugar Estate, Ethiopia. PLoS One. 2014; 9(10): p. e109793.

[13] Jayarani, K., T. Sandhya Rani, and K. Jayaranjani, Intestinal parasitic infections in pre school and school going children from rural area in Puducherry. Current Research in Microbiology and Biotechnology. 2014; 2(4): p. 406-409.

[14] Mengistu M, S.T., Torben W, Terefe A, Kassa T, Hailu A:, Human intestinal schistosomiasis in communities living near three rivers of Jimma town, south Western Ethiopia. Ethiopian journal of health sciences. 2011; 21(2): p. 111-118.

[15] Montresor A, C.D., Hall A, Bundy DAP,Savioli L,. Guidelines for the evaluation of soil-transmitted helminthiasis and schistosomiasis at community level. Geneva: World Health Organization.1998; p. 1-48.

[16] USAID/ETHIOPIA, End of the Project Evaluation for the Urban Health Extension Program. online. 2014.

[17] Yami A, M.Y., and Kebede S., Prevalence and predictors of intestinal helminthiasis among school children in jimma zone; a cross-sectional study. Ethiopian journal of health sciences. 2011; 21(3).

[18] Rim HJ, C.J., Min DR, Cho SY, Eom KS, Hong SJ, et al.,, Prevalence of intestinal parasite infections on a national scale among primary schoolchildren in Laos. Parasitology research. 2003; 91(4): p. 267-272.

[19] Sayyari AA, I.F., Bagheri Yazdi SA, Karami H, Yaghoobi M,. , Prevalence of intestinal parasitic infections in the Islamic Republic of Iran. 2005.

[20] Tadesse D, T.A., Impact of irrigation on the prevalence of intestinal parasite infections with emphasis on schistosomiasis in Hintallo-Wejerat, North Ethiopia. Ethiopian Journal of Health Science. 2008; 18(2): p. 6.

[21] Marothi Y, S.B., Prevalence of intestinal parasites at Ujjain, Madhya Pradesh, India: Five-year study. Afr J Microbiol Res. 2011; 5(18): p. 2711-2714.

[22] Chhakda T, M.S., Socheat D, Odermatt P., Intestinal parasites in school-aged children in villages bordering Tonle Sap Lake, Cambodia. Southeast Asian journal of tropical medicine and public health. 2006; 37(5): p. 859.

[23] Fekadu D, B.P., Amha K. , Hookworm species distribution among school children in Asendabo Town, Jimma Zone, South West Ethiopia. Ethiopian Journal of Health Science. , 2008. 18: p. 4.

[24] Serkadis D, A.W., Nejat J, Zeleke M., Soil transmitted helminths and associated factors among schoolchildren in government and private primary school in Jimma town, southwest Ethiopia. Ethiop J Health Sciences. 2013; 23(3).

[25] Megbaru Al, A.H, Gessessew B. , Prevalence of intestinal schistosomiasis and soil transmitted helminthiasis among primary school children in Umolante district, South Ethiopia. Clinical Medicine Research. 2014; 3(6): p. 7.

[26] Abate, A., et al., Cross-Sectional Study on the Prevalence of Intestinal Parasites and Associated Risk Factors in Teda Health Centre, Northwest Ethiopia. ISRN Parasitology, 2013. 2013.

[27] Asrat A, T.D., Alemayehu W,, Prevalence and risk factors of intestinal parasites among Delgi school children, North Gondar, Ethiopia. Journal of Parasitology and Vector Biology. 2011; 3(5): p. 6.

[28] Zeleke M, S.M., Mio A, Johannes B, Josef V, Bruno L. , Comparison of individual and pooled stool samples for the assessment of soil-transmitted helminth infection intensity and drug efficacy. . PLoS neglected tropical diseases. 2013; 7(5). 